\title{
Management of CML blast crisis and the role of SCT
}

\author{
Rüdiger Hehlmann \\ Universität Heidelberg, Germany
}

Prof. Dr. Dr. h.c. R. Hehlmann, Medizinische Fakultät

Mannheim der Universität Heidelberg, Pettenkoferstr. 22, 68169 Mannheim, Germany
Phone: +49 (0) 621-383-69-31;

Fax: +49 (0)621-383-69-32

E-mail: R.Hehlmann@urz.uni-heidelberg.de

\section{Resume}

Incidence of BC is low, with $5.6 \%$ after 8 years.

Survival after BC not much improved with TKI.

Treatment of BC in CML study IV is heterogeneous as expected without specific recommendations in the study protocol.
No recommendation of a specific drug treatment is possible.

Transplantation carries the best long term prognosis in BC.

Allo SCT always recommended in BC by ELN.

\section{Keywords}

chronic myeloid leukemia, blast crisis, stem cell transplantation, experimental treatment.
Progression of chronic myeloid leukemia to blast crisis (CML $\mathrm{BC}$ ) is still a phenomenon that is only incompletely understood. In up to $90 \%$ of BC patients, additional chromosomal aberrations (ACA) are reported. In up to $77 \%$ mutations are detected by means of deep genome sequencing. In gene expression profiles blast crisis appears as a disease distinct from CML. Treatment continues to be mostly unsuccessful unless allo SCT is offered. Treatment failure indicates point of no return. Additional chromosomal aberrations (ACA) are associated with CML blast crisis, being non-random in chromosomal distribution. Major route ACA are $+8(34 \%)$, $+\mathrm{Ph}(30 \%), \mathrm{i}(17 \mathrm{q})(20 \%),+19$ (13\%), +21 (7\%), +17 (5\%), monosomy 7 (5\%). Temporal order of secondary changes varies, preferred pathway $\mathrm{i}(17 \mathrm{q}),+8,+\mathrm{Ph},+19$.

There exist certain diagnostic criteria for CML acceleration phase (CML AP) and BC. I.e., AP is characterized by 15 $29 \%$ blasts in blood or marrow (WHO criteria, 10 - 19\%); > $20 \%$ basophils in blood; persistent thrombocytopenia unrelated to therapy; unresponsiveness to therapy. The blast crisis is registered by $>30 \%$ blasts in blood or marrow (WHO,
20\%); extramedullary blastic infiltrates; varying degrees of fever, anemia, splenomegaly, leukocytosis. Different means of blast crisis prevention are considered while analyzing the studies of 1983 to 2013. Special attention is drawn to tyrosine kinase inhibitors (TKIs, imatinib, nilotinib, dasatinib) in blast crisis. Introduction of imatinib led to a small increase of patient survival (up to $20 \%$ ). Most long term survivors are those that have been transplanted $(28 / 39$, ca. $72 \%)$. European Leukemia Net (ELN) presented analysis of allo-HSCT results in CML blast crisis.

Several approaches are considered. I.e., HSCT should be always used as a second-line treatment in blast phase, irrespective of the response to TKIs. It is recommended "always" in accelerated phase, if the response to TKI is less than optimal, and, as third-line therapy, if the response to second-line TKI is less than optimal. The value and the meaning of "Always" depend on transplant risk (age, comorbidities, performance status, donor, etc.).

Induction of $2^{\text {nd }}$ chronic phase $(\mathrm{CP})$, or remission with TKI 
alone or in combination with intensive chemotherapy was studied at various regimens by several study groups, i.e.: IM 800 mg + VCR + Dexa (DIV), Rea et al., 2006 (LBC) $(\mathrm{n}=13)$ $\mathrm{IM}+$ mitoxantrone / etoposide + AraC (Frühauf et al., 2007), (MBC) $(\mathrm{n}=16) \mathrm{IM}+$ Dauno-AraC (Deau et al., 2011) $(\mathrm{n}=36)$ Dasatinib + FLAG-IDA (Milojkovic et al., 2012), $(\mathrm{n}=4)$ SCT with HLA identical related or matched unrelated donor was performed at EBMT score of 0 to 4 , and standard conditioning. Maintenance TKI treatment conditions after SCT have been studied by several groups (Hehlmann et al., Blood, 2012; Rea et al., 2006; Frühauf et al., Cancer 2007; Deau et al., 2011; Milojkovic et al., 2012). Application of AlloHSCT as a $2^{\text {nd }}$ line therapy was studied by CML Study IV (84 patients). HSCT after imatinib failure in $1^{\text {st }} \mathrm{CP}$ was accompanied by a $94 \%$ survival rate, whereas HSCT performed in advanced phase of CML yielded a 3-year survival of 59\% (Saußele et al., 2010).
Similar results were published by Oyekunle et al. (2013).

Experience with blast crisis treatment was summarized for CML Study IV ( $\mathrm{n}=1551$ patients). The main regimen was imatinib $400 \mathrm{mg}+\mathrm{AraC}$ or intensive chemotherapy. A special assessment of CML study IV concerned the survival rates after allo-HSCT in blast crisis dependent on pretreatment, but patient numbers were too small for firm conclusions.

Comparisons were made for myeloid vs. lymphoid BC. Treatment of lymphoid type BC was associated somewhat longer median survival than myeloid-type BC. Further data analysis is in progress. Investigational trials in this field are underway (Table 1).

\begin{tabular}{|l|l|}
\hline Biological effects & Drugs/compounds \\
\hline PP2A activation & Fingolimod (FTY720) \\
\hline & SET antagonist OP449 \\
\hline Self renewal of LSC & CIP2A inhibitor \\
\hline & BCL6 + TK inhibitors \\
\hline & HIFla inhibitor \\
\hline Activation of apoptosis & Smoothened inhibitors (in combination with TKI) \\
\hline & Jak2 inhibitor (in combination with TKI) \\
\hline & BCL2-inhibitor ABT-737 \\
\hline 0thers & Triptolide \\
\hline & Dual-kinase inhibitor 0N044580 \\
\hline & $\begin{array}{l}\text { MEK inhibitor PD184352 + } \\
\text { farnesyltransferase inhibitor BMS-214662 }\end{array}$ \\
\hline
\end{tabular}

Abbreviations, TKI: tyrosine kinase inhibitor; PP2A: protein phosphatase 2A; LSC: leukemia stem cells; MEK: mitogen-activated protein kinase; HIF: hypoxia induced factor; HDAC: histone deacetylase; Hsp: heat shock protein.

Table 1. Investigational approaches in CML blast crisis

\section{References}

1. Deau B, Nicolini FE, Guilhot J, Huguet F, Guerci A, Legros L, Pautas C, Berthou C, Guyotat D, Cony-Makhoul P, Gardembas M, Michallet M, Hayette S, Cayuela JM, Weiss IR, Réa D, Castaigne S, Mahon FX, Guilhot F, Rousselot P. The addition of daunorubicin to imatinib mesylate in combination with cytarabine improves the response rate and the survival of patients with myeloid blast crisis chronic myelogenous leukemia (AFR01 study). Leuk Res. 2011; 35(6):777-82.
2. Fruehauf S, Topaly J, Buss EC, Fischer T, Ottmann OG, Emmerich B, Müller MC, Schuld P, Balleisen L, Hehlmann $\mathrm{R}$, Ho AD, Hochhaus A. Imatinib combined with mitoxantrone/etoposide and cytarabine is an effective induction therapy for patients with chronic myeloid leukemia in myeloid blast crisis. Cancer. 2007; 109(8):1543

3. Hehlmann R. How I treat CML blast crisis. Blood. 2012; 120(4): 737-747. 